\title{
Moralische Charakterbildung, Leadership Excellence und Corporate Character
}

\author{
JOSEF WIELAND ${ }^{* *}$
}

In der Tradition der europäischen Unternehmensethik ist der Charakter der Akteure ein grundlegender und alles dominierender Zurechnungsmodus für tugendhaftes Handeln. Die psychologische und völkerkundliche Diskussion einer „Charakterkunde" in der zweiten Hälfte des 19. Jahrhunderts hat diese Tradition vor allem in Deutschland durch nationalsozialistische und antisemitische Interpretationen desavouiert. Aber es gibt noch einen weiteren, aus theoretischer Sicht entscheidenderen Grund für den Traditionsbruch. Aristoteles theoretische Diskussion der ethischen Tugenden und die Praxis des „Ehrbaren Kaufmanns“ lassen hier wenig Zweifel. In funktional differenzierten Gesellschaften ist Charakter ein schwieriger Begriff, weil er sich nicht mehr auf den Status von Bürgern, sondern als Forderung nach Integrität als offener Rechtsbegriff auf Funktionsträger bezieht. Der Artikel diskutiert vor diesem Hintergrund und mit der Linse der Governanceethik die mit den psychologischen Verhaltenswissenschaften und der experimentellen Ökonomik gegebenen Optionen eines strukturalistischen Charakterbegriffs.

Schlagwörter: Charakterbildung, Leadership Excellence, Corporate Character, Governanceethik

\section{Moral Character Formation, Leadership Excellence and Corporate Character}

In the tradition of European business ethics the character of the actors is a fundamental and dominant mode for the attribution of virtuous action. The psychological and ethnological discussion of a "science of character" in the second balf of the 19th century brought this tradition into bad repute, above all in Germany as a result of the National Socialist and anti-Semitic interpretations. But there is a further reason for this break, with tradition, which is decisive from a theoretical point of view. Aristotle's theoretical discussion of the ethical virtues and the practice of the "bonourable merchant" allow little doubt about this. In functionally differentiated societies character is a difficult concept because it no longer refers to the status of citizens but is an open legal concept demanding integrity from functionaries. Against this background the article discusses the options for a structuralist concept of character provided by the psychological bebavioural sciences and experimental economics as seen through the lens of Governance Ethics.

Keyzords: Character Formation, Leadership Excellence, Corporate Character, Governance Ethics

\footnotetext{
* Beitrag eingereicht am 12.11.2013, nach doppelt verdecktem Gutachterverfahren überarbeitete Fassung angenommen am 27.01.2014.

** Prof. Dr. habil. Josef Wieland, Leadership Excellence Institute Zeppelin (LEIZ)/Zeppelin Universität, Am Seemooser Horn 20, 88045 Friedrichshafen, Tel.: +49 (0)7541-6009-2261, Fax: +49 (0)7541-6009-1299, E-Mail: josef.wieland@zu.de, Forschungsschwerpunkte: Institutional Economics, Organisational Governance, Integrity Management \& Transcultural Leadership.
} 


\section{Unternehmensethik und die Frage nach dem Charakter der Akteure}

\subsection{Der Stand der Diskussion}

Wenn wir die letzten drei Jahrzehnte der unternehmensethischen Diskussion summarisch und ohne Anspruch auf Vollständigkeit daraufhin analysieren, welche Erträge sie für die Praxis der Unternehmen erbracht hat, dann hat diese Bilanz einiges zu bieten (vgl. Werhane/Freeman 2006; Aßländer 2011; Zsolnai 2013). Die philosophische Ethik hat durch Begriffsklärungen und die Entwicklung analytischer Konzeptionen, die - wenngleich sie sich auf sehr unterschiedliche philosophische Traditionen und Schulen beziehen - Kriterien der Evaluierung und Legitimierung ökonomischen Entscheidens und Handelns bereitgestellt, die heute in allen internationalen Standards guten unternehmerischen Verhaltens und gesellschaftlicher Verantwortung eine wesentliche Rolle spielen. So etwa die Prinzipien des Diskurses, der Inklusion aller relevanten Stakeholder oder der Fairness. Ebenfalls ist es ein Verdienst der philosophischen Diskussion, die Zielkonflikte und Dilemmata ethischen Entscheidens in der Wirtschaft zu thematisieren und nach Wegen ihrer Bewältigung durch rationale Analyse und Verfahren zu suchen (vgl. für diese Diskussion im deutschsprachigen Raum Ulrich 2008; Palazzo/Scherer 2006; Steinmann/Löhr 1994).

Das bisher für die philosophische Diskussion Gesagte trifft in modifizierter Weise auch auf die ökonomische Ethik zu, wobei hier ökonomische Modelle und Analyseverfahren angewandt werden, die die Anreizstruktur der Dilemmasituation und der Akteure im Zentrum der Ermöglichung moralischen Handelns in der Wirtschaft sehen. Dahinter steht ein Aufklärungsanspruch, der voll und ganz auf das Erklärungspotenzial ökonomischer Rationalität und die Anreizwirkung möglicher Win-winSituationen setzt. Konsequenterweise gilt das Interesse an Dilemmasituationen weniger dem Feld ethischer Dilemmata, sondern dem Gefangenendilemma und möglichen Anreizstrategien seiner Vermeidung und Überwindung. Auch diesem Ansatz ist es gelungen, strukturierend in die öffentliche wirtschaftsethische Diskussion einzugreifen und dort die Leistungsfähigkeit des entwickelten kategorialen Instrumentenkastens unter Beweis zu stellen (vgl. Homann/Blome-Drees 1992; Homann/Pies 2000; Homann/Suchanek 2005).

Die Governanceethik ${ }^{1}$, die in den Bereich der Institutionen- und Organisationsethik fällt, hat sich auf die theoretische Analyse der Integration der Unternehmensethik in eine institutionenökonomisch fundierte Theorie der Firma und der Corporate Governance $^{2}$ konzentriert. Einer der Schwerpunkte der Governanceethik war und ist die praktische Entwicklung effektiver und adaptiv effizienter Governancestrukturen zur Implementierung moralischer Ansprüche an wirtschaftliche, nationale und globale Transaktionen. Unternehmensethische Implementierungsstrategien können sich auf individuelle Selbstbindung, formale und informale Institutionen der Gesellschaft und Organisationsstrukturen als Governancemechanismen stützen, deren spezifische Kombination und Integration über die Erfolgsaussichten ethischen Handelns in der Wirtschaft entscheiden. Werte-Management-Systeme, Integrity- und Compliance-

$1 \quad$ Zur Einführung siehe Wieland (2007/1999).

2 Vgl. zum Stand der unternehmensethischen Corporate-Governance-Diskussion Brink (2011).

zfwu $15 / 3$ (2014), 376-397 
Management-Systeme und transkulturelle Leadership-Entwicklung sind auf diese Weise, einschließlich der dazugehörenden Instrumente, entwickelt und standardisiert worden und gehören heute zu den zunehmend akzeptierten Standards guter Managementpraxis (vgl. Wieland 2004; 2010c; 2010d; 2010e; 2014a).

Die Beiträge der verhaltenswissenschaftlichen und sozialpsychologischen Behavioural Business Ethics ${ }^{3}$ zur unternehmensethischen Diskussion beziehen sich einerseits auf die Prozesse individueller ethischer Urteilsbildung und moralischen Verhaltens, andererseits, und zu einem nicht geringen Teil, auf die Analyse der motivationalen und emotionalen Entstehungsbedingungen von deviantem Verhalten. Dabei geht es praktisch um alle Formen der Wirtschaftskriminalität, die hauptsächlich erklärt werden über Typen der Selbst- und Fremdlegitimation von Tätern (vgl. Veiga et al. 2004; Coleman 2005; Hübscher 2010; Wieland 2010b) und die Auslösungsmechanismen für dolose Handlungen (vgl. grundlegend Cressey 1971; Treviño et al. 1999; Weaver/Treviño 1999; Smith et al. 2007; Wieland 2010b). In der experimentellen Ökonomik steht die Erklärung von sozialem/unsozialem und ethischem/unethischem Verhalten, etwa Folter, Lüge, Sabotage sowie Empathie, Fürsorglichkeit, Fairness und so weiter, in gegebenen Anreizsituationen im Vordergrund, die in Abgrenzung vom selbstinteressierten „Homo oeconomicus" einen eigenständigen Zugang zu ökonomischer Forschung ermöglicht (vgl. Fehr/Schmidt 1999; Harbring/Irlenbusch 2011; Irlenbusch/Neschen 2013; Kenning/Wobker 2013). Ebenfalls zu erwähnen ist für die unternehmensethische Diskussion der Globalisierung von Wertschöpfungsketten die Durchführung verhaltenswissenschaftlicher Experimente im „Cross-Cultural“-Kontext (vgl. Henrich et al. 2005; Herrmann et al. 2008).

\subsection{Moralpsycholgische Desiderata}

Die theoretischen und praktischen Erträge der soeben referierten verschiedenen Forschungsansätze für die unternehmensethische Diskussion sind also beachtlich und es gibt Grund zu der Annahme, dass sich diese Entwicklung kontinuieren wird. Wenn wir nach Forschungsdesideraten und Praxisdefizienzen der Wirtschafts- und Unternehmensethik Ausschau halten, dann fällt auf, dass sich die bisher etablierten Forschungsstrategien häufig auf die Ausarbeitung und Anwendung rationaler, individueller und organisationaler Kommunikations- und Entscheidungsstrategien für Unternehmensethik beziehen, seltener jedoch, mit Ausnahme der Behavioural Business Ethics, auf die moralpsychologischen Voraussetzungen und Fähigkeiten der wirtschafts- und unternehmensethischen Akteure als Komponente gelingender moralischer Urteilsbildung und Praxis aufmerksam gemacht haben. ${ }^{4}$ Eine gewisse Ausnahme bildet die erzichungswissenschaftlich ansetzende Forschung zur Entwicklung indivi-

\footnotetext{
3 Vgl. für eine Übersicht Treviño et al. (2006); Plott/Smith (2008); Cropanzano/Stein (2009); Salvador/Folger (2009); Wieland (2010a); Sadler-Smith (2012).

4 Dies gilt für die deutsche Diskussion. Für die lebhafte angelsächsische Diskussion wären beispielhaft zu erwähnen: Damon (2002); Paine (1991); Treviño (1992); Werhane (1999; 2007); aus kommunitarischer Perspektive siehe Etzioni (2002). Hier scheinen auch kulturell bedingte Unterschiede vorzuliegen. Während etwa 90 Prozent der Befragten U.S.-amerikanischen Manager angeben, das zu „ethical leadership“ in erster Linie „Charakter" eine Rolle spielt, sind es in Deutschland nur 50 Prozent (vgl. Resick et al. 2011: 443).
} 
dueller moralischer Kompetenz und Urteilsbildung. Hier gibt es eine Reihe von Beiträgen, die sich etwa unter dem Gesichtspunkt der beruflichen Bildung mit motivationalen Fragestellungen auseinandersetzen (vgl. Minnameier 2005; 2011; Minnameier et al. 1999; Minnameier/Schmidt 2013; Lind 1985; 2002; 2009 sowie die Beiträge in Edelstein et al. 1993), deren Anschluss an die wirtschafts- und unternehmensethische Diskussion bisher aber nur selten gesucht wird. (vgl. dazu vor allem Retzmann/Grammes 2011; Retzmann 1994; 2005; 2009). Obgleich diese Diskussion einen wesentlichen Beitrag zur empirisch-inhaltlichen Füllung der Charakterentwicklung im Sinne der Entstehung und Entwicklung moralischer Kompetenz und Urteilsbildung leisten könnte, werden ihre Ergebnisse in der folgenden Diskussion keine Berücksichtigung finden. Hier wird es in der Hauptsache um die Herauspräparierung der moralpsychologischen Dimension des Zusammenspiels von individueller, organisatorischer und gesellschaftlicher Charakterbildung, also um eine strukturalistische Theorie des Charakters gehen. Dies ist angesichts der alteuropäischen philosophischen und unternehmerischen Tradition, also etwa Aristoteles ${ }^{\circ}$ grundlegende Unterscheidung zwischen Verstandes- und ethischen Charaktertugenden (vgl. Aristoteles 1967: 1103a14) und des geschichtlichen Einflusses des Leitbildes eines „Ehrbaren Kaufmanns“ auf die Entwicklung des internationalen Handels ${ }^{5}$, erstaunlich oder eben vielleicht auch genau deshalb nicht. Denn die Tugendlehren des Aristoteles und des „Ehrbaren Kaufmanns" sind keine rationalen ethischen Analyseinstrumente, sondern zielen auf gelungene Lebensführung, die, über ethische Ansprüche weit hinausgehend, eine allgemein und immer schon gültige Norm, die Pflicht zur Kultivierung menschlicher Existenz, zugrunde legt. So gehörten zum Kanon des „Ehrbaren Kaufmanns“ praktische Grundfähigkeiten (Mathematik, Schriftverkehr, Warenkunde etc.), tugendhaftes Verhalten (Anstand, Redlichkeit, Keuschheit, kein Glücksspiel etc.), Normen der Lebensführung (guter gesellschaftlicher Umgang, mäßiger Alkoholgenuss etc.) und Charaktereigenschaften (Toleranz, Freundlichkeit, Vertrauenswürdigkeit, gute Erscheinung, kein Geschwätz, Mäßigung, Ordnung etc.). Gelegentliche zeitgenössische Versuche, den „Ehrbaren Kaufmann“" als freiwilligen Verhaltensstandard gegen staatliche Regulierung ins Feld zu führen, haben allerdings wenig historische Evidenz für sich, da diese immer schon in formale, private und öffentliche Regulierungen und Erzwingungsmechanismen eingebettet waren (vgl. Kadens 2012). Heute werden die Berufungen auf „Ehre“ als Handlungsmoral in eher vormodern geprägten Interaktionsverhältnissen gepflegt, während sie in den westlichen Gesellschaften als unangemessene Eingriffe in das Recht auf individuelle Lebensgestaltung markiert und ausgesondert werden. Allerdings liefert der $\$$ Sec.316.10 der „General Requirements for Naturalization" des „U.S. Citizenship and Immigration Services" eine mehrstufige Definition des „Good Moral Character" (vgl. USCIS 2013), die sich kaum von frühneuzeitlichen Vorstellungen unterscheidet - ebenso die Prüfung zur Niederlassung als Rechtsanwalt in Kalifornien (vgl. TSBOC 2013) -, ohne dass dem ein bürgergesellschaftliches Erzwingungsinstrumentarium entspricht. In genau diesem Sinne gehört der „Ehrbare Kaufmann" auch zur Satzung der deutschen Industrie- und Handelskammern (vgl. DIHK 2013) und kann damit gegebenenfalls zum Referenzpunkt rechtlicher Bewer-

5 Für einen Überblick siehe Klink (2008); Schwalbach/Klink (2012) sowie Kadens (2012) und die dort verarbeitete Literatur.

zfwu 15/3 (2014), 376-397 
tung unternehmerischen Handelns werden. Insgesamt bietet daher die Figur des „Ehrbaren Kaufmanns“ nur wenige Anknüpfungspunkte für eine wirtschafts- und unternehmensethische Charaktertheorie.

Da scheint die Referenz auf die aristotelische Tradition zunächst aussichtsreicher. Verstandestugenden erwirbt man, so Aristoteles, durch Studium und Bildung, ethische Charaktertugenden durch Übung und Gewohnheit. Für die Idee, dass ein guter Charakter sich beim theoretischen Studium bildet, hat Aristoteles nur nachsichtigen Spott übrig: „Ohne zu handeln, dürfte keiner wohl jemals tugendhaft werden“ (Aristoteles 1967: 1105b10). Das ist seine Zusammenfassung der Unternehmensethik als praktisches Handeln, ,denn indem wir im Geschäftsverkehr den Menschen gegenüber handeln, werden wir, die einen gerecht, die anderen ungerecht (...) (Aristoteles 1967: 1103b15). Unternehmensethik, so könnte man aristotelisch formulieren, ist von Anfang an eine ,praktiké" und zwar eine solche, die sich auf die in der Polis immer schon eingelassenen und unveränderlichen Grundprinzipien menschlichen Zusammenlebens bezieht (vgl. Höffe 1987; 2008).

Der alteuropäische und frühneuzeitliche Begriff des ethischen Charakters entspricht der Praxis hierarchisch-stratifizierter Gesellschaften, die zur ethischen Orientierung und Entscheidung in moralisch strittigen Angelegenheiten der Person an der Spitze der Hierarchie, also der Statuszurechnung, bedürfen (vgl. Luhmann 1994). Es gibt daher exzellente Gründe dafür, dass sich die zeitgenössische unternehmensethische Diskussion bisher eher zurückhaltend auf diese Version des Charakters bezogen hat. Denn funktional differenzierte Gesellschaften rechnen nicht auf Status, sondern auf Funktionserfüllung und Systeme zu (vgl. Wieland 2005: 11ff.). Die an anderer Stelle (vgl. Wieland 2014b) von mir ausführlich erörterte Einforderung der Integrität und der Vorbildrolle des Führungspersonals von Unternehmen durch die World Bank Group Integrity Compliance Guidelines, dem „Principle 2“ der „Appropriate Measures" des UK Bribery Act und die Einforderung von „Effective Compliance and Ethics Programs" im $\ 8$ B2.1 der US Sentencing Guidelines zielen auf die Effektivitätssicherung von Compliance-Management-Systemen und richten sich nicht an moralische Respektspersonen der Gesellschaft, sondern an ökonomische und rechtliche Funktionsträger (vgl. Wieland 2013). Integrität ist daher in der modernen juristischen und unternehmensethischen Diskussion ein funktionales Äquivalent für den moralischen Charakter, aber nicht mit diesem identisch, weil Integrität sich nicht auf die Gesamtheit der Lebensführung bezieht, sondern auf die „Risk based Due Diligence“ und "Compliance" einer Agenturfunktion in einem wirtschaftlichen Kontext. Integrität wird damit zu einem unbestimmten Rechtsbegriff, dessen inhaltliche Auffüllung dem gesellschaftlichen Diskurs, dem gerichtlichen Urteil oder aber dem IntegrityManagement-System von Unternehmen überlassen wird. Darauf werden wir später ausführlicher zurückkommen.

Die Ausfüllung der moralischen Pflicht zur Kultivierung der Lebensführung als Selbstkultivierung, also des in der modernen Gesellschaft abgespaltenen Aspekts des alteuropäischen Charakterbegriffs, kann auf diese Weise individualisiert und privatisiert, bei Bedarf aber auch ökonomisiert werden. Gestützt auf ein ganzes Arsenal von Moden, Trends, „,must haves“, Benimm-Schulen, Ess- und Trinkkulturen und so weiter wird daraus dann nicht nur eine geldwerte Dienstleistung der Lebensmittel-, Un- 
terhaltungs-, Mode-, Ratgeber- und Freizeitindustrie, sondern eben auch eine auf Temporalisierung angelegte gesellschaftliche Charakternorm, nämlich: mit der Zeit zu gehen. Eine unternehmensethische Diskussion entsteht daraus jedoch nicht.

\section{3 „Wille zur Sittlichkeit"?}

Damit könnte die Diskussion über den ethischen Charakter ökonomischer Akteure aus unternehmensethischer Sicht beendet sein, wenn da nicht die oben erwähnten Forschungsdesiderate und Praxisdefizienzen wären. Diese bestehen ja darin, dass rationale Analyse-, Argumentations- und Entscheidungssysteme und klug konstruierte und instrumentalisierte ökonomische Anreiz- und Managementsysteme nur einen begrenzten Einfluss auf die Motivation, tatsächlich ethisch zu handeln, haben. Nur weil ethisches Handeln rational begründbar ist oder ein formales Werte-ManagementSystem in einem Unternehmen existiert, wird kaum jemand in einer durch die ökonomische Logik strukturierten Umwelt tatsächlich ethisch handeln. Rationale Begründungen haben nur eine geringe motivationale Kraft. Erziehung, das Streben nach Selbstkonsistenz, moralische Anreize und Emotionen wie Empörung, Mitleid, Sympathie scheinen da eher als Trigger für moralisches Handeln infrage zu kommen (vgl. exemplarisch Gaudine/Thorne 2001; Marquardt/Hoeger 2009; Mencl/May 2009; Priddat 2010). Wir wissen nicht nur nicht genug darüber, warum Akteure in einer gegebenen Situation moralisch handeln, sondern wir wissen auch zu wenig darüber, und hier folgen wir Lynn Sharp Paine, warum Akteure sich ,entschließen“, eine gegebene Situation als moralisch codierte wahrzunehmen, sich „entschließen“, sich mit dieser Situation zu beschäftigen und zu akzeptieren, dass eine persönliche Entscheidung zum Handeln und nicht die $\mathrm{Ab}$ - und Verdrängung eines lästigen, unpopulären oder unlösbaren Problems ansteht. Mit anderen Worten: Rationale Analyse- und Entscheidungskonstrukte und Managementsysteme sind notwendige, aber nicht hinreichende Bedingungen moralischer Praxis. Die Frage, warum Menschen sich ,entschließen", moralisch zu handeln, und welche kognitiven und emotionalen Prozesse darin involviert sind, ist für die weitere theoretische Entwicklung der Unternehmensethik von entscheidender Bedeutung. Gibt es so etwas wie einen „Willen zur Sittlichkeit" und könnte es nicht dieser sein, der den moralischen Charakter ausmacht? Paine scheint in diese Richtung zu argumentieren, wenn sie feststellt, dass Instrumente, Managementsysteme und Analyse- und Entscheidungsmodelle ,must be seen in the context of an overall conception of character" (Paine 1991: 71).

„Moreover, only someone who cares to use the tools, who recognizes when they are appropriate, and who wants to do what they recommend will benefit from careful instruction in how to use them" (Paine 1991: 72).

Die Relevanz dieser Überlegung wird durch die bereits erwähnte jüngere Entwicklung in der unternehmensethischen Praxis unterstrichen, nämlich, die Enthaftungswirkung und die Effektivität von Compliance-Management-Systemen an den Nachweis der Integrität der Führungskräfte eines Unternehmens zu binden. Reine „Legal Compliance" ist damit kein Nachweis mehr, dass das Management seinen Sorgfaltspflichten

6 So jedenfalls Konfuzius: ,Sobald ich die Sittlichkeit wünsche, so ist diese Sittlichkeit da“ (Konfuzius 2008: 29). 
und Verantwortlichkeiten nachgekommen ist, sondern dieser Nachweis kann allenfalls über eine „Integrity and Values driven Compliance“" erbracht werden. Integrität als funktionales Äquivalent des ,,moralischen Charakters“ von Führungskräften der Wirtschaft und dessen gerichtsverwertbarer (und damit auch intersubjektiv gelingender) Nachweis sind damit zum legalen und moralischen Anspruch der Gesellschaft an die Wirtschaft und ihrer Akteure avanciert. Es ist also Zeit für Philosophie und Wissenschaft, sich damit zu beschäftigen, mit welchem Phänomen wir es hier, unter den Bedingungen der Moderne, zu tun haben. Und damit sind wir bei einer verhaltenswissenschaftlichen Diskussion des Charakters, der wir uns nun zuwenden wollen. Wir stimmen also Paine zu, wenn sie feststellt:

„The habits of thought, the concerns, and the commitments that lead one to see a situation as requiring action or decision play a role in this picture of choice, which focuses on deliberative rationality" (Paine 1991: 70).

\section{Drei Ebenen der ethischen Charakterbildung: Individuum, Orga- nisation, Gesellschaft}

\subsection{Statischer und dynamischer Charakterbegriff}

Eine conditio sine qua non, um den Prozess der ethischen Charakterbildung einer unternehmensethischen Erörterung zugänglich zu machen, besteht darin, den Charakter des Charakters zu klären.

$\mathrm{Da}$ ist zunächst die doppelte Bedeutung des Wortes, nämlich Charakter als Kennzeichnung einer Person und Charakter als ethische Tugend. Dass jemand als schlechter, guter, zuverlässiger, dickköpfiger oder aufbrausender Charakter beschrieben werden kann, bezieht sich auf bestimmte Persönlichkeitsmerkmale, die sich nicht unbedingt einem ethischen Urteil verdanken, wohl aber auf Tugenden - beziehungsweise deren Abwesenheit - verweisen, die für gesellschaftliche Inklusion notwendig sind. Diese liegen ebenfalls der schon erwähnten Vorstellung eines „Ehrbaren Kaufmanns“ zugrunde, mit dem das aufkommende Bürgertum seine Identität und Gesellschaftsfähigkeit analog zum Adel überhaupt erst konstituieren konnte und von dem bereits erwähnt wurde, dass es diesem nicht allein und nicht einmal in erster Linie um die Repräsentanz einer ethischen Qualität ging, sondern um die Konstituierung einer ehrbaren, also im Geschäft handlungs- und kooperationsfähigen, weil vertrauenswürdigen Person. Wer in dieser Welt seine Ehre verliert, verliert nicht allein seine moralische Dignität, sondern mit seiner Kreditwürdigkeit auch seinen Platz in der Gesellschaft (vgl. Klink 2008: 69). Dieses statische Verständnis des Charakterbegriffs als Markierung einer Persönlichkeit wird uns für die aktuelle unternehmensethische Diskussion nicht weiter beschäftigen.

Charakter als ethische Tugend kann ebenfalls in zwei Bedeutungen verstanden werden und zwar in einer statischen und einer dynamischen Interpretation. Von Aristoteles stammt der Satz, der die Charakterdiskussion bis auf den heutigen Tag prägt, nämlich:

„Es kommt nicht wenig darauf an, ob man gleich von Jugend auf an dies oder jenes gewöhnt wird: es kommt vieles darauf an, ja sogar alles" (Aristoteles 1967: 1103b25). 
„Was Hänschen nicht lernt, lernt Hans nimmermehr"; das ist eine der stehenden Weisheiten der Volkskultur, und ich halte es für möglich, dass auch Aristoteles hier nur wiedergibt, was "Common Sense“ im Athen des 4. Jahrhunderts v. Chr. war. Denn diese statische Interpretation des Charakters, nämlich, dass die Weichen in frühester Kindheit nahezu unabänderlich gestellt werden, widerspricht seiner theoretisch getriebenen Unterscheidung, dass der ethische Charakter sich nur im Vollzug von Alltagspraxis bildet, bewährt und reift, also einem dynamischen Lern- und Entwicklungsprozess unterliegt, denn in der Praxis des Menschen gibt es "nichts Stabiles“ (Aristoteles 1967: 1104a5). In der Welt eines statischen Charakters ist Unternehmensethik überflüssig, weil sie unwirksam ist. ${ }^{7}$ In der dynamischen Welt der Charakterbildung und Charakterformation als praktischem Lernprozess tritt neben die individuelle Ebene der Charakterbildung die Ebene der Organisation und der Gesellschaft. Organisationen und gesellschaftliche Institutionen sind Vollzugsmechanismen wirtschaftlicher und gesellschaftlicher Praxis und daher genuine Bestandteile der Charakterbildung als einem Prozess. Der Psychoanalytiker Erich Fromm argumentiert in diese Richtung, wenn er darauf hinweist, ,dass die eigentliche Basis des Charakters (...) in den verschiedenen Arten, in denen sich ein Mensch zur Welt in Beziehung setzt" (Fromm 1995: 54), also im „Vollzug des Lebens“ (Fromm 1995: 54), liegt. In die gleiche Richtung argumentiert Richard Bondi, wenn er knapp notiert: „Character is the self in relation" (Bondi 1984: 214).

Die Orientierungen des Lebensvollzugs, so Fromm, entstammen einerseits den Gemeinschaften des Individuums, also etwa der Gesellschaft oder den Organisationen, denen es zugehört, andererseits aber auch seinen individuellen (und darin eingeschlossen auch allen kindlichen) Prägungen. Fromm unterscheidet den „Gesellschaftscharakter" sowie den ,individuellen Charakter" (Fromm 1995: 56) und wir wollen aus der Sicht der Governanceethik den „organisationalen Charakter“ oder den „Corporate Character" hinzufügen. Die Dynamik der Charakterbildung ergibt sich in diesem Theoriedesign aus dem Zusammenspiel von Gesellschafts-, Organisations- und Individualcharakter, aus den formalen und informalen Institutionen einer Gesellschaft, der Governance von Organisationen und der Integrität ihrer Akteure. Im Folgenden werde ich versuchen, Ansätze zu einer strukturalistischen Theorie des Charakters zu entwickeln, strukturalistisch in dem Sinne, dass die konstitutiven Elemente des Charakters - die Gesellschaft, die Organisation und das Individuum - nur als kontextuelle Struktur, deren je einzelner Einfluss und Wirkung aus ihrer Gesamtheit als wechselwirkende und integrierende Struktur emergiert, verstanden werden können. Charakter wird dann als Struktur von Strukturen und von dieser Struktur her verstanden, also aus ihrer Eigenschaft als Governancestruktur. Das schließt nicht aus, sondern ein, dass die theoretische Analyse und Diskussion des individuellen, organisatorischen und gesellschaftlichen Charakters je einzeln geführt werden kann und auch muss. Aber der Prozess der Charakterbildung lässt sich nur als Emergenz einer Governancestruktur verstehen.

Wir wollen uns aus unternehmensethischer Sicht für den weiteren Gang der Argumentation mit Hilfe der theoretischen Unterscheidungen zwischen Individual-, Orga-

7 Diesen Punkt macht zu Recht Paine (vgl. Paine: 1991: 69).

zfwu $15 / 3$ (2014), 376-397 
nisations- und Gesellschaftscharakter auf die Erarbeitung einer dynamischen und strukturalistischen Konzeptionierung des Charakters von ökonomischen Akteuren als moralische Fähigkeit im Vollzug von wirtschaftlichen Transaktionen konzentrieren.

\subsection{Der individuelle Charakter}

Wir sollten uns für eine unternehmensethische Diskussion zunächst um eine inhaltliche Auffüllung des individuellen Charakterbegriffs bemühen, die deren Anschlussfähigkeit an eine verhaltenswissenschaftliche Diskussion herstellt. ${ }^{8}$ Der Begriff der Identität scheint sich für diese strukturelle Kopplung zu eignen, da er sich sowohl auf Individuen als auch auf Organisationen beziehen lässt und verschiedenen theoretischen Disziplinen zugänglich ist.

Michele Mangini verweist darauf, dass die Verbindung von Psychologie und Ethik im Hinblick auf die individuelle Charakterbildung fruchtbar gemacht werden sollte (vgl. Mangini 2000: 81), und operationalisiert diese Einsicht über die Begriffe der Identität und Personalität. Während für ihn die Identität die innere Balance von Kohärenz, Vitalität, Tiefe und Reife einer Person bezeichnet (vgl. Mangini 2000: 84), so ist Personalität als ein distinktes und über die Zeit stabiles Set von Qualitäten zu verstehen, etwa Fairness, Fürsorglichkeit, Ehrlichkeit, Wahrhaftigkeit und Generosität.

„It is normal patterns of thought and action, especially with respect to concerns and commitments, that make up who a person is, his or her character"(Mangini 2000: 87).

Die Sozialisierung und kontinuierliche soziale Anerkennung von folgenorientierter Compliance mit Regeln, Respekt vor sich selbst und anderen und Sympathie für andere um der anderen willen, also Emotion und Vernunft, Motivation und Handeln, sind konstituierende Elemente des Charakters von Personen (vgl. Mangini 2000: 87f.). Diese Definition hat in Zeiten globaler Kooperation den Vorzug, dass sie auf Verallgemeinerungsfähigkeit hin ausgelegt ist. Denn in der Tat ist es so, dass gelingende moralische Transkulturalität, so Mangini, eine gemeinsame Vorstellung über einen guten Charakter voraussetzt (vgl. Mangini 2000: 88).

Paine geht in ihren Überlegungen ebenfalls davon aus, dass substanzielle individuelle Charakterentwicklung möglich und normal ist. Die Anwendung und die Erfahrungen aus der Anwendung von Werten lösen Lernprozesse aus, die vorhandene Charakterzüge stärken oder schwächen können (vgl. Paine 1991: 74). Zentral für die inhaltliche Auffüllung des personalen Charakterbegriffs ist für sie der Begriff der Integrität, der für Ehrlichkeit, Fairness, Einhaltung von Verpflichtungen, Respekt vor Menschen und Eigentum, die Ablehnung arglistiger Täuschung und die Fähigkeit zur kritischen Selbstreflexion steht. Auf der Akteurs-Ebene werden diese Prinzipien durch Emotionen, Dispositionen und Einstellungen für Handlungen aktiviert, aber nicht durch ökonomische Kalkulation.

8 Vgl, zur Übersicht über den Stand der Diskussion Homiak (2011). Aber auch hier bleibt es bei dem eingangs konstatierten Forschungsdesiderat einer moralpsychologischen Füllung des Charakterbegriffs, der uns in diesem Artikel als mögliche Brücke zur wirtschafts- und unternehmensethischen Diskussion beschäftigt. 
„Although the environment in which she works will be an important influence, there are certain personal capacities that are critical: the capacity for ethical sensibility; the capacity for ethical reasoning; the capacity for ethical conduct; the capacity for ethical leadership. These capacities are the backbone of character" (Paine 1991: 77).

Die Sensibilität, die moralische Herausforderung, die in einer wirtschaftlichen Transaktion steckt, überhaupt zu erkennen und für sich selbst als Handlungsaufforderung zu akzeptieren, verlangt nach, und darauf hat Patricia Werhane (Werhane 1999; 2007) hingewiesen, „Moral Imagination“. Diese startet mit einem Ereignis, das eine moralische Dimension im Betrachter wachruft, schafft Distanz zur Reflexion, was eigentlich getan werden sollte und was die Konsequenzen einer solchen Entscheidung wären. In diesem Sinne ist „Moral Imagination“ produktiv und kreativ und nicht bloße Reflexion (vgl. Werhane 1999: $101 \mathrm{ff}$.). Sie entwickelt sich über drei Stadien, nämlich i) die „Reproductive Imagination“ der Situation, ii) die „Productive Imagination“, die diese Situation auf neue Möglichkeiten untersucht, und schließlich iii) die „Creative Imagination", die nach neuen Handlungsmöglichkeiten sucht (vgl. Werhane 2007: 85). In einer etwas anderen Version sind die drei Phasen:
„Beginning with awareness of the particular, moral imagination (...) includes
(1) awareness of the character, context, situation, event, and dilemma at issue;
(2) awareness of the script or schema function in that context and role rela- tionship entailed in that context, and (3) awareness of possible moral conflict or dilemmas that might arise" (Werhane 1999: 103).

\begin{abstract}
„Moral Imagination“ ist demnach eng verbunden mit der Schaffung von Aufmerksamkeit, die ihrerseits wiederum geschult und geschärft werden kann durch das Erzählen moralischer Geschichten (vgl. Werhane 2007: 86ff.). Genauso, wie das Erzählen von Märchen über das Gute und das Böse nicht wenig Einfluss auf die moralische Sozialisation von Kindern hat, weil hier die Imaginierung von moralischem Gelingen angeregt und zugleich asymmetriert (,, und wenn sie nicht gestorben sind, dann leben sie noch heute") wird, gilt dies allgemein für die Herausbildung von Personalität und Charakterentwicklung. Es sind Geschichten und weniger Argumente, die moralische Emotionen und Engagement triggern. Diesen Punkt hat Richard A. Graham ebenfalls stark gemacht:
\end{abstract}

"It is storytelling, in particular, that provides the basis for moral imagination" (Graham 2003: 159).

Wer sich nicht vorstellen kann, dass Business Ethics möglich ist und kein Widerspruch in sich selbst darstellt, der wird zwar vielleicht aus Furcht vor Bestrafung ,legal compliant" sein können, aber kein Integrity Management wirksam implementieren können. Aber man sollte sich davor hüten, Imagination und Kalkulation gegeneinander auszuspielen:

„Whether a person acts on his or her judgment of what is fair or good in a
particular situation, depends largely on one's sense of obligation to fulfill the
responsibility at hand when weighted against conflicting responsibilities. The
decision to act depends also on one's self-assessment of one's ability to act
competently to succeed in carrying out the responsibility. Judgment tells what

zfwu 15/3 (2014), 376-397 
ought to be done; imagination assesses what reasonably can be done: Ought implies can"(Graham 2003: 161).

Wenn wir die hier referierte Diskussion zusammenfassen, dann bieten sich aus verhaltenswissenschaftlicher Sicht vier Faktoren an, die einen signifikanten Einfluss auf die individuelle Charakterbildung haben:

(1) Awareness, als vorhandene, gewollte und geschulte Aufmerksamkeit für die moralische Dimension ökonomischer Transaktionen,

(2) Perception, als die Fähigkeit, diese moralische Dimension zu identifizieren und zu verstehen,

(3) Acceptance, als das innere Einverständnis, die aus dieser Dimension folgende Handlungsaufforderung anzuerkennen,

(4) Commitment, als Verpflichtung, diese Handlungsaufforderung auf sich selbst zu beziehen und sie nicht an andere zu delegieren.

\subsection{Der organisationale Charakter}

Wir wollen uns nun vor dem Hintergrund dieser vier Faktoren der Erörterung des Organisationscharakters zuwenden. Wird dieser allein und ohne Rückgriff auf Tugendhaftigkeit erklärt, endet man notwendig bei methodologischen Schwierigkeiten. So etwa Geoff Moore, der durch eine tautologische Definition von Tugend und Charakter versucht, den Begriff des „Corporate Character“ zu schärfen:

„A virtuous corporate character is the seat of the virtues necessary for a corporation to engage in practices with excellence" (Moore 2005: 661).

Dass ein tugendhafter Charakter der Ausgangspunkt aller Tugenden ist, die zu tugendhafter Praxis führen, ist kaum zu bestreiten, erklärt aber relativ wenig.

Edith Penrose betont die Rolle der Imagination bei der Allokation aller unternehmerischen Ressourcen, also nicht nur der moralischen, und in der Tat kann das Einführen und Zulassen moralischer Ressourcen in den Prozess der Wertschöpfung nicht geschehen ohne die generelle Imaginationsfähigkeit des Managements, wo und in welcher wirtschaftlichen oder gesellschaftlichen Hinsicht dies notwendig zu geschehen hat und mit Aussicht auf Erfolg geschehen kann. Penrose nutzt dafür die Unterscheidung zwischen Organisation und Umwelt:

„In order to focus attention on the crucial role to a firm's 'inherited' resources, the environment is treated, in the first instance, as an 'image' in the entrepreneur's mind of the possibilities and restrictions with which he is confronted, for it is, after all, such an 'image' which in fact determines a man's behaviour; whether experience confirms expectations is another story" (Penrose 1995/1959: 29).

Ich werde im Verlauf der weiteren Diskussion noch einmal ausführlicher auf den $\mathrm{Zu}$ sammenhang von Imagination und Handeln zurückkommen, aber das Folgende sollte hier kurz angemerkt werden: Die Fähigkeit zur Imagination gehört grundlegend zum Charakter eines Unternehmers, oder allgemeiner einer Führungskraft, als moralische Fähigkeit. Aber Leadership Excellence ergibt sich erst durch die Einbettung des individuellen Charakters in eine entsprechende Organisations- und Führungskultur und 
den operativen Mechanismen eines Werte-Management-Systems (vgl. McCoy 1985; Wieland 2004; 2011), deren Gesamtheit den Organisationscharakter bestimmt. Individuelle moralische Fähigkeit ist Bestandteil von Leadership Excellence, aber diese muss integriert werden in die entsprechenden ökonomischen und organisatorischen Fähigkeiten, die erst in diesem Verbund, in diesem Netz voll zur Geltung gelangen können. Governance ist in der Governanceethik definiert als die Mechanismen der Führung, des Managements und des Monitorings von Transaktionen und unterscheidet sich damit wesentlich von dem herkömmlichen, auf den Aspekt des Monitorings verengten Begriffsverständnis der Governance (vgl. Jensen/Meckling 1976; Fama/Jensen 1983a; 1983b und Wieland 2014a, Kapitel 1, I). Aber es ist eben die Struktur der Integration von Führung, Management und Monitoring, die die Effizienz und Effektivität der gesamten Governancestruktur bestimmt, die als die Repräsentanz der Einheit der Differenz dieser drei Komponenten verstanden werden kann. So, wie es der Governanceethik nie allein um ethische oder ökonomische, sondern um moralökonomische Transaktionen geht, so gilt auch für die Bestimmung vom Charakter als moralökonomische Fähigkeit in der Führung von Unternehmen, dass diese nur als Einheit der Differenz von Ökonomie und Moral, Struktur und Motivation verstanden, konzipiert und realisiert werden kann. In den Worten von Chester L. Barnard":

„Purposeful cooperation is possible only within certain limits of a structural character [...] The work of cooperation is not a work of leadership, but of organization as a whole" (Barnard 1968/1938: 259).

Um diesen strukturellen Aspekt besser zu verstehen, verbleiben wir zunächst noch bei der Diskussion der moralischen Charakterdimension von Organisationen. Organisations- und Führungskultur und operative Werte-Management-Systeme können die Bildung von Aufmerksamkeit für moralische Fragen fördern, ignorieren oder blockieren. Gleiches gilt für die Faktoren der Wahrnehmung, der Akzeptanz und der Selbstverpflichtung. Dieser Zusammenhang ist wesentlich für die Erklärung sowohl individueller als auch organisatorischer Charakterbildung, da beide Prozesse rekursiv vernetzt sind. Sie können sich wechselseitig fördern, ignorieren oder blockieren.

\subsection{Der gesellschaftliche Charakter}

Wir haben bereits bei Fromm gelernt, dass der gesellschaftliche Charakter sich aus der Präge- und Steuerungswirkung gesellschaftlicher Institutionen wie dem Recht, der Familie, der Schule oder dem Markt ergibt. Institutionen sind Elemente des Vollzugs des Lebens und können daher wie die Organisationen der Gesellschaft einen fördernden, ignorierenden oder blockierenden Einfluss auf die individuelle Charakterbildung haben, genauso aber auch umgekehrt. Es muss zur Veranschaulichung dieses Zusammenhangs an dieser Stelle ein kurzer Rückverweis auf die weiter vorne geführte Diskussion über die rechtlichen Grundlagen des Integrity- und Compliance-Managements reichen, die überhaupt erst die Frage nach dem Charakter der Führungskräfte der Wirtschaft als eine unternehmensethische Fragestellung auf die Agenda der aktuellen gesellschaftlichen Diskussion gesetzt haben. Integrität, Tone from the top und Vor-

\footnotetext{
9 Vgl. für eine auf Barnard aufbauende Konzeption von Organisationscharakter Selznick (1957).
} 
bildrolle sind die einschlägigen Codewörter dieser Diskussion um Leadership Excellence, deren Effektivität nur im strukturellen Kontext bewertet kann.

Wir fassen die in diesem Kapitel geführte Diskussion über die drei Ebenen des Charakters in dem folgenden Schaubild zusammen, das die Dynamik und den Prozess der Charakterbildung illustrieren soll.

\begin{tabular}{|l|l|l|l|}
\hline & $\begin{array}{c}\text { Individuum } \\
{[1,0,-1]}\end{array}$ & $\begin{array}{c}\text { Organisation } \\
{[1,0,-1]}\end{array}$ & $\begin{array}{c}\text { Gesellschaft } \\
{[1,0,-1]}\end{array}$ \\
\hline \hline Awareness & $\ldots$ & $\ldots$ & $\ldots$ \\
\hline \hline Perception & $\ldots$ & $\ldots$ & $\ldots$ \\
\hline \hline Acceptance & $\ldots$ & $\ldots$ & $\ldots$ \\
\hline \hline Commitment & $\ldots$ & $\ldots$ & $\ldots$ \\
\hline
\end{tabular}

$[1$ = fördernd; $0=$ ignorierend; $-1=$ blockierend $]$

Abbildung 1: Dynamike der Charakterbildung

(Quelle: eigene Darstellung)

Es ist die bereits erwähnte Interaktion der konstitutiven Ebenen des Charakters (das Individuum, die Organisation und die Institution) mit den Faktoren des Charakters (awareness, perception, acceptance und committment), die zu einer strukturalistischen Erklärung des Prozesses der Charakterbildung führt. Charakterformation vollzieht sich in einem strukturellen Kontext wechselwirkender Ebenen und Faktoren. Charakterbildung als dynamischer Prozess kann durch die Governance der Ebenen und Entwicklung der Faktoren in eine Richtung entwickelt, aber nicht determiniert werden. Auch dieser Gesichtspunkt ist für die Bewertung von Integrity- und ComplianceManagement von grundlegender Bedeutung, da nur die Gesamtheit der Governancestruktur und dann erst die Analyse ihre einzelnen Faktoren die Wirksamkeit des moralischen Charakters erklärt.

\section{Leadership Excellence und Corporate Character}

\subsection{Intersektorale Konflikte und Dilemmata}

Wir wollen zum Abschluss das bereits weiter vorne angedeutete Verhältnis von Corporate Character und Leadership Excellence diskutieren, von dem die bisherige Diskussion gezeigt hat, dass es von einiger unternehmensethischer Bedeutung ist.

Der Wille und die Fähigkeit zu moralischer Reflexion als individuelle Charaktereigenschaft beziehen sich einerseits auf den professionellen Umgang mit Werte- und Interessenkonflikten und moralischen Dilemmata. Umgang meint nicht immer Lösung, denn moralische Dilemmata kann man nicht lösen, sonst wären sie keine Dilemmata, 
sondern sie verlangen nach einer Entscheidung. Es sind oft "Tragic Choices" (vgl. Calabresi/Bobbitt 1978). Die Kraft, diese Entscheidungen zu treffen und dabei eventuell auch Schuld auf sich zu laden, ist eine besondere Fähigkeit des individuellen moralischen Charakters und basiert auf der starren Plastizität seiner Werteorienticrung. Anders hingegen verhält es sich mit Werte- und Interessenkonflikten, die einer rationalen Bewertung und Entscheidung zugänglich sind, die auf einer Abwägung von Vor- und Nachteilen beruhen. Dabei spielen, anders als im Dilemma, die eigene Nutzenvorstellung und die Folgenkalkulation der eigenen Handlung eine wesentliche Rolle, die in einem Wertekonflikt gerade nicht aufgegeben, sondern gefordert werden muss. Wesentliche Aufgabe von organisatorischem Wertemanagement und von strategischem normativen Management, damit ist die Existenz eines Code of Ethics, Code of Conduct, von Verfahren und Leitlinien und Monitoring-Prozessen zu deren Umsetzung gemeint, ist dafür zu sorgen, dass es so wenige Konflikte und Dilemmata wie möglich für den individuellen Entscheider gibt. Sie sind Ausdruck und Vollzug des Charakters einer Organisation, eines kollektiven Akteurs, des Unternehmens und zielen auf die Aktivierung der vier Faktoren der Charakterformation. Ihnen geht es einerseits um die Vermeidung von Konflikt- und Dilemmasituationen sowie die Prävention nicht akzeptablen Verhaltens, andererseits geht es um die Sicherstellung der Fähigkeit einer Organisation, im Einklang mit ihren moralischen Überzeugungen und ihren ökonomischen Zielen zu agieren. Individuelle Motivation wird an dieser Stelle durch Struktur funktional äquivalent unterstützt, entwickelt und gegebenenfalls auch ersetzt, die Wirkungsfähigkeit des individuellen Charakters wird als moralökonomische Fähigkeit durch den Organisationscharakter erhöht. Umgekehrt gilt ebenfalls, dass die moralökonomischen Ambitionen eines kollektiven ökonomischen Akteurs nur realisiert werden können durch die Bereitschaft und Fähigkeit des individuellen Akteurs, sowohl moralisch als auch ökonomisch zu handeln. Individueller und organisatorischer Charakter als moralökonomische Fähigkeit stehen demnach grundsätzlich in einem Steigerungsverhältnis und Simultanität ist daher ihr Wesenszug. Eine substitutive Beziehung der beiden kann nur die Ausnahme und nicht die Regel sein.

\subsection{Rekursivität von Führung und Charakter}

Andererseits ist der Wille und die Fähigkeit zu moralökonomischer Reflexion notwendig, um die vielfältigen moralischen und ökonomischen Anforderungen verschiedener Handlungsbereiche des Unternehmens und der darin involvierten Stakeholder-Interessen zu verstehen und zu entscheiden. Auch diese müssen wahrgenommen, identifiziert, akzeptiert und als selbstverpflichtend erlebt werden. Voraussetzung dafür ist, dass die Führungskräfte der Wirtschaft über Polylingualität verfügen, also die Fähigkeit, die Sprachspiele der verschiedenen Stakeholder möglichst nah an ihrem Eigenwert zu rekonstruieren und durch intersektorales Management, das heißt unter Berücksichtigung der verschiedenen gesellschaftlichen und organisatorischen Ressourcen und Interessen, in den Wertschöpfungsprozess des Unternehmens zu integrieren. Ich stimme Paine zu, wenn sie feststellt:

„Today's business leader must be able to formulate ethically sound positions on numerous public issues affecting business. The capacity for this sort of reasoning can be strengthened by examining characteristic patterns and fallacies in arguments and by practice in formulating and defending arguments for various positions" (Paine 1991: 80). 
Neben die Fähigkeit zur Imagination treten daher auch die der Polylingualität und Intersektoralität als Aktivierungsvoraussetzungen der entwickelten vier Faktoren der Charakterformation.

Dabei kann es nicht nur um die eigenen Fähigkeiten der Führungskraft gehen, sondern auch darum, die Vermittlung dieser Fähigkeiten an andere, etwa Mitarbeiter, zu beeinflussen und zu kreieren. Barnard hat diese Fähigkeit zum Wesensmerkmal von Leadership Excellence erklärt:

„The creative aspect of executive responsibility is the highest exemplification of responsibility" (Barnard 1968/1938: 281).

Ohne individuelle moralische Ressourcen, und Charakter ist eine solche Ressource, und organisatorische Werte-Management-Systeme, mit ihren vielfältigen Mechanismen, ist das aber, so auch Paine, nicht wirkungsvoll möglich (vgl. Paine 1991: 83). Genau darin liegt die Logik der bereits mehrfach erwähnten rechtlichen Regulierungen zum Integritäts- und Compliance-Management, die die Integrität der Unternehmensführung zu einem rechtlich relevanten Tatbestand gemacht haben. Aus Sicht einer Führungskraft unterstützt Integrity Management die Sicherstellung und Entfaltung ihrer eigenen Integrität und der Integrität ihrer Mitarbeiter.

Leadership Excellence ergibt sich vor dem Hintergrund der bisher geführten Diskussion nicht aus der Definition von ethischen Führungsstilen und Führungstypen. So verdienstvoll die mikroskopisch und häufig ad hoc differenzierende Literatur zur Unterscheidung von Stilen und Typen der Führung auch sein mag (vgl. Stone et al. 2004; Conger 2012; Walumbwa/Wernsing 2012), aus ihr lässt sich wenig lernen darüber, worin genau die Exzellenz in der ethischen Führung von Unternehmen liegt und an welchen Faktoren sie sich entscheidet. Aus der Sicht der Governanceethik ist Leadership Excellence das Ergebnis des Zusammenspiels von Motivation und Struktur, der Generierung, Aktivierung und Integration von informalen (etwa Unternehmenskultur) und formalen (Leitlinien und Verfahren), von gesellschaftlichen (Standards) und privaten (Integrity- und Compliance-Management-Systemen) Governancestrukturen durch Management einer Organisation.

An dieser Stelle lohnt es sich, auf die „Fuzzy Logic“, die Unschärfe von Werten in der Handlungsbestimmung im Anwendungskontext und die bereits weiter vorne erwähnte Rolle von „Corporate Stories" zu verweisen. Was der Wert „Wahrhaftigkeit" im Prinzip meint, ist allgemein unstrittig, welche Handlung daraus allerdings in einer bestimmten Situation erwartet wird, kann lokal unklar und strittig sein und ist es auch. Moralisches Handeln aus moralischen Prinzipien ist nur sehr selten selbstevident. Die praktische Scharfstellung der Unschärfe von Werten, die alle vier Faktoren der Charakterbildung berührt, läuft über alle Komponenten individueller, organisationaler und institutioneller Leadership Excellence. "Tone from the Top", "Corporate Stories", organisationale Verhaltensrichtlinien und gesellschaftliche Wertekulturen zielen auf die situationelle Klärung der Bedeutung und praktischen Konsequenzen von moralischen Prinzipien und Werten, deren Funktionen Identitätsbildung, Schaffung eines Wahrnehmungsrahmens und normative ethische Orientierung sind. Mit dieser situationellen Spezifikation wird die „Fuzzy Logic“ von Werten limitiert und damit überhaupt erst die Voraussetzung für moralökonomisches Verhalten und Handeln geschaffen. Die Voraussetzung moralökonomischen Verhaltens wäre demnach eine zweifache: die 
gelingende Spezifikation der situationalen Unschärfe von Werten durch einen Handelnden und das Vorhandensein der Charaktereigenschaft, die moralische Situation als solche überhaupt wahrzunehmen. Aus der Sicht der Behavioral Business Ethics ist daher der Problemvorwurf moralischen Handelns ein doppelter: Erstens, die genaue Definition dessen, was in einem empirischen Kontext genau die aus Wertvorstellungen und ethischen Prinzipien folgende Handlungserwartung ist (situationaler Faktor) und, zweitens, die kognitive und emotionale Fähigkeit und Bereitschaft, moralisch Handeln zu wollen, also Verantwortung zu übernehmen (motivationaler Faktor). Liegt eine der beiden Bedingungen nicht vor, kommt es zur Verdrängung und Nichthandlung. Das folgende Schaubild zeigt den dabei sich herausbildenden Mechanismus der rekursiven Selbststabilisierung von Leadership Excellence und Charakter, bei der die Beziehung zwischen Werten und Charakter eine über Praxis und Kommunikation vermittelte ist und deren Drehscheibe die Exzellenz der Führung einer Organisation und ihrer Governancestrukturen ist.

Werte

$F_{u z Z y^{\prime} \log i c}$

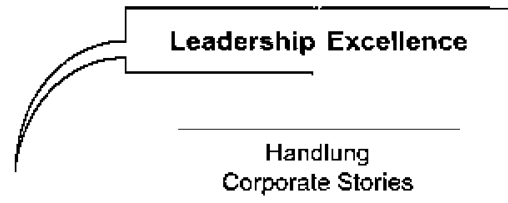

Corporate Stories

Charakter
Ethische Nometivitat

Moralokonamische Amwendung

Personale, organisationale und institutionele Govemancestrukturen

Moralökonomische Handung \&

Kommunikation

Morakkonomische Fähigkent

\section{Abbildung 2: Rekursivität von Leadership Excellence und Charakter} (Quelle: eigene Darstellung)

Die Abbildung zeigt, dass moralische Charakterbildung und Leadership Excellence keine statischen Zustände sind, sondern ein Entwicklungsprozess, dessen Ziel die Ermöglichung ökonomischer und gesellschaftlicher Wertschöpfung und Kooperation zum wechselseitigen Vorteil ist. Dies ist nur als kontinuierlicher Lernprozess der personalen und organisationalen Charakterbildung möglich. Charakter bezeichnet in diesem Kontext keine metaphysische, vererbte, unveränderliche oder höchst subjektive Qualität, sondern die Bereitschaft und Fähigkeit, nach Verantwortlichkeit, nach Exzellenz in der Führung zu streben.

\section{Einige Schlussfolgerungen}

Ich habe versucht dafür zu argumentieren, dass die weitere fruchtbare Entwicklung der Unternehmensethik durch den Einbezug von verhaltenswissenschaftlicher For- 
schung gefördert werden kann. Ich habe dies am Thema der ethischen Charakterbildung und Charakterentwicklung diskutiert, wobei ich Charakter als Wille und Fähigkeit von Personen und Organisationen moralisch zu handeln definiert und ein Stück weit entwickelt habe. Der Einbezug von Befunden aus verschiedenen Bereichen der verhaltenswissenschaftlichen Forschung und ihre Kombination mit den Ergebnissen der Forschung der Governanceethik waren die methodische Grundlage der Erörterung.

Ein aus meiner Sicht wesentliches Ergebnis der Diskussion ist, dass sich die Unternehmensethik nicht nur für deviantes Verhalten (Wirtschaftskriminalität, Verletzung von Sozialstandards, Bruch von Menschenrechten, unmoralisches Managerverhalten und so weiter), also die Verletzung von moralischen Standards, interessieren sollte, sondern stärker auch für moralisches Verhalten. Was sind die Bedingungen moralischer Imagination? Wie ist der Mechanismus beschaffen, der die „Fuzzy Logic“ von Werten mit den umfassenden moralischen Konzepten von Werten verbindet? Was sind gute „Moral Stories" und wie werden sie erfolgreich kommuniziert? Wie funktionieren die Wahrnehmungsprozesse für moralische Situationen, wie die dazugehörenden Entscheidungsprozesse? Welche Beschaffenheit haben moralische Anreize und wie ist ihre Interaktion mit ökonomischen Anreizen? Was sind die Merkmale von „Moral Education“ in Theorie und Praxis? Wie ist das Verhältnis von moralischer Motivation und organisatorischer Struktur? Wie funktionieren die dazugehörenden "Crowding-out-" und simultanen Steigerungsprozesse? Was sind die Bedingungen für die Emergenz einer global akzeptierten normativen Ordnung? Wie hängen interkulturelle und transkulturelle Ethiken, moralische Differenz und Gemeinsamkeit zusammen?

Diese Auflistung von Forschungsfragen ist nicht vollständig, sondern nur beispielhaft. Was damit kurz und gut gesagt werden sollte, ist, dass wir uns stärker dafür interessieren sollten, warum und entlang welcher Mechanismen Menschen moralisch handeln, und nicht primär dafür, warum sie es nicht tun. Moralisches Handeln ist nicht selbstverständlich (vgl. Schlink 2009) und Birger Priddat hat zu Recht auf den weitreichenden Unterschied von „moral behaviour“ und „moral action“ im Hinblick auf den Charakter des „moral entrepreneurs“ (vgl. Priddat 2013: 137f.) aufmerksam gemacht. Die Unternehmensethik kann nicht nur in dieser Hinsicht viel lernen bei den Verhaltenswissenschaften.

\section{Literaturverzeichnis}

Aristoteles (1967): Die Nikomachische Ethik, München-Zürich: Artemis.

Aßländer, M. S. (Hrsg.) (2011): Handbuch der Wirtschafts- und Unternehmensethik, Stuttgart: Metzler.

Barnard, C. J. (1968/1938): The Functions of the Executive, Cambridge: Harvard University Press.

Brink, A. (Hrsg.) (2011): Corporate Governance and Business Ethics, Berlin: Springer.

Bondi, R. (1984): The Elements of Character, in: The Journal of Religious Ethics, Vol. 12/No. 2, 201-218.

Calabresi, G./Bobbitt, P. (1979): Tragic Choices, New York: W. W. Norton \& Company. 
Coleman, J. W. (2005): The Criminal Elite. The Sociology of White-Collar Crime, New York: Worth Publishers.

Conger, J. A. (2012): Charismatic Leadership, in: Rumsey, M. G. (Ed.): The Oxford Handbook of Leadership, Oxford: Oxford University Press, 376-391.

Cressey, D. R. (1971): Other People's Money. A Study of Social Psychology of Embezzlement, Belmont: Wadsworth.

Cropanzano, R./Stein, J. H. (2009): Organizational Justice and Behavioural Ethics. Promises and Prospects, in: Business Ethics Quarterly, Vol. 19/No. 2, 155-233.

Damon, W. (Ed.) (2002): Bringing a New Era in Character Education, Stanford: Hoover Institution Press.

Deutsche Industrie und Handelskammer (DIHK) (2013): Ehrbarer Kaufmann. Link: http://www.dihk.de/themenfelder/recht-steuern/rechtspolitik/grundsatzthemen/ ehrbarer-kaufmann (zuletzt abgerufen am 03.08.2013).

Edelstein, W./Nunner-Winkler, G./Noam, G. (Eds.) (1993): Moral und Person, Frankfurt a. M.: Suhrkamp.

Etrioni, A. (2002): A Communitarian Position on Character Education, in: Damon, W. (Ed.): Bringing a New Era in Character Education, Stanford: Hoover Institution Press.

Fama, E. F./Jensen, M. C. (1983a): Separation of Ownership and Control, in: Journal of Law and Economics, Vol. 26/No. 2, 301-325.

Fama, E. F./Jensen, M. C. (1983b): Agency Problems and Residual Claims, in: Journal of Law and Economics, Vol. 26/No. 2, 327-349.

Febr, E./Schmidt, K. M. (1999): A Theory of Fairness, Competition and Cooperation, in: Quarterly Journal of Economics, Vol. 114/No. 3, 817-868.

Fromm, E. (1995): Psychoanalyse und Ethik. Bausteine zu einer humanistischen Charakterologie, München: dtv.

Gaudine, A./Thorne, L. (2001): Emotion and Ethical Decision-Making in Organizations, in: Journal of Business Ethics, Vol. 31/No. 2, 175-187.

Grabam, R. A. (2003): Imagining Self and Society, in: McLean, G. F./Knowles, R. (Eds.): Moral Imagination in Personal Formation and Character Development, Washington D. C.: Council for Research in Values and Philosophy, 157-165.

Harbring, C./Irlenbusch, B. (2011): Sabotage in Tournaments. Evidence from a Laboratory Experiment, in: Management Science, Vol. 57, 611-627.

Henrich, J./Boyd, R./Bowles, S./Camerer, C./Fehr, E./Gintis, H./McElreath, R./Alward, M./Barr, A./Ensminger, J./ Smith-Henrich, N./Hill, K./Gil-White, F./Gurven, M./Marlowe, F. W./Patton, J. Q./Tracer, D. (2005): "Economic Man" in Cross-cultural Perspective: Behavioral Experiments in 15 Small-scale Societies, in: Behavioral and Brain Sciences, Vol. 28/No. 6, 795-815.

Hermann, B./Tböni, C./Gäcbter, S. (2008): Antisocial Punishment Across Societies, in: Science, Vol. 319/No. 5868, 1362-1367.

Hoffe, O. (1987): Politische Gerechtigkeit. Grundlegung einer kritischen Philosophie von Recht und Staat, Frankfurt a. M.: Suhrkamp.

Höffe, O. (2008): Praktische Philosophie. Das Modell des Aristoteles, Berlin: Akademie.

Homann, K./Pies, I. (2000): Wirtschaftsethik und Ordnungspolitik. Die Rolle wissenschaftlicher Aufklärung, in: Leipold, H./Pies, I. (Hrsg.): Ordnungstheorie und Ordnungspolitik. Konzeptionen und Entwicklungsperspektiven, Stuttgart: Lucius \& Lucius, 329-346.

Homann, K./Blome-Drees, F. (1992): Wirtschafts- und Unternehmensethik, Göttingen: Vandenhoeck \& Ruprecht. 
Homann, K./Suchanek, Andreas (2005): Ökonomik. Eine Einführung, 2. Auflage. Tübingen: Mohr Siebeck.

Homiak, M. (2011): Moral Character, in: Zalta, E. N. (Ed.): The Stanford Encyclopedia of Philosophy. Link: URL = http://plato.stanford.edu/archives/spr2011/entries/ moral-character/ (zuletzt abgerufen am 03.08.2013).

Hübscher, M.C. (2010): Lucifer in between. Governance und schmutzige Praxis. Die Psychologie der Moralverdrängung und das Recht auf Rechtfertigung, in: Wieland, J. (Hrsg.): Behavioural Business Ethics. Psychologie, Neuroökonomik und Governanceethik, Marburg: Metropolis, 63-102.

Irlenbusch, B./Neschen, A. (2013): Zur Bedeutung der Experimentellen Wirtschaftsforschung für die Wirtschaftsethik - Dargestellt an Beiträgen zur Korruptionsforschung, im Erscheinen.

Jensen, M. C./Meckling, W. H. (1976): Theory of the Firm. Managerial Behavior, Agency Cost and Ownership Structure, in: Journal of Financial Economics, Vol. 3/No. 4, 305-360.

Kadens, E. (2012): The Myth of the Customary Law Merchant, in: Texas Law Review, Vol. 90/No. 1153, 1153-1206.

Kenning, P./Wobker, I. (2013): Ist der „mündige Verbraucher" eine Fiktion? Ein kritischer Beitrag zum aktuellen Stand der Diskussion um das Verbraucherleitbild in den Wirtschaftswissenschaften und der Wirtschaftspolitik, in: Zeitschrift für Wirtschafts- und Unternehmensethik, Jg. 14/Heft 2, 282-300.

Klink, D. (2008): Der Ehrbare Kaufmann - Das ursprüngliche Leitbild der Betriebswirtschaftslehre und individuelle Grundlage für die CSR-Forschung, in: Zeitschrift für Betriebswirtschaft, Heft. 3, 57-79.

Konfurius (2008): Die Lehren des Konfuzius, Neu-Isenburg: Wunderkammer.

Küng, H./Leisinger, K. M./Wieland, J. (2010): Manifest Globales Wirtschaftsethos, München: dtv.

Lind, G. (1985): Inhalt und Struktur des moralischen Urteilens. Theoretische, methodologische und empirische Untersuchung zur Moral- und Demokratiekompetenz bei Studierenden. Dissertationsschrift, Universität Konstanz.

Lind, G. (2000): Ist Moral lehrbar?, Berlin: Logos-Verlag.

Lind, G. (2009): Moral ist lehrbar. Handbuch zur Theorie und Praxis moralischer und demokratischer Bildung, München: Oldenburg Verlag.

Lubmann, N. (1994): Die Wirtschaft der Gesellschaft, Frankfurt a. M.: Suhrkamp.

Mangini, M. (2000): Character and Well-being. Towards an Ethics of Character, in: Philosophy and Social Criticism, Vol, 26/No. 2, 79-98.

Marquardt, N./Hoeger, R. (2009): The Effect of Implicit Moral Attitudes on Managerial Decision-Making. An Implicit Social Cognition Approach, in: Journal of Business Ethics, Vol. 85/No. 2, 157-171.

MoCoy, C. S. (1985): Management of Values, Boston: HarperCollins.

Mencl, J./May, D. R. (2009): The Effects of Proximity and Empathy on Ethical DecisionMaking. An Exploratory Investigation, in: Journal of Business Ethics, Vol. 85/No. 2, $201-226$.

Minnameier, G./Beck, K./Heinrichs, K./Parcbe-Kawik, K. (1999): Homogeneity of Moral Judgement? Apprentices solving business conflicts, in: Journal of Moral Education, Vol. 28/No. 4, 429-443.

Minnameier, G. (2005): Wer Moral hat, hat die Qual, in: Zeitschrift für Berufs- und Wirtschaftspädagogik, Jg. 101/Heft 1, 19-42.

Minnameier, G. (2011): Situationsspezifität moralischen Denkens und Handelns. Befunde, Erklärungen und didaktische Orientierungen, in: Zlatkin-Troitschanskaia, O. (Hrsg.): 
Stationen empirischer Bildungsforschung. Traditionslinien und Perspektiven, Wiesbaden: VS, 107-122

Minnameier, G./Scbmidt, S. (2013): Situational Moral Adjustment and the Happy Cictimizer, in: European Journal of Developmental Psychology, Vol. 10/No. 2, 253-268.

Moore, G. (2005): Corporate Character. Modern Virtue Ethics and the Virtuous Corporation, in: Business Ethics Quarterly, Vol. 15/No. 4, 659-685.

Paine, L. S. (1991): Ethics as Character Development: Reflections on the Objective of Ethics Education in: Freeman, E. R. (Ed.): Business Ethics. The State of the Art, New York: Oxford University Press, 67-86.

Palazo, G./Scherer, A. G. (2006): Corporate Legitimacy as Deliberation: A Communicative Framework, in: Journal of Business Ethics, Vol. 66/No. 1, 71-88.

Penrose, E. (1995/1959): The Theory of the Growth of the Firm, New York: Oxford University Press.

Plott, C. R./Smith, V.L. (2008): Handbook of Experimental Economics Results 1, Amsterdam: North Holland.

Priddat, B. P. (2010): Ökonomie, Moral, Emotion - the affective turn in economics in: Wieland, J. (Hrsg.): Behavioural Business Ethics. Psychologie, Neuroökonomik und Governanceethik, Marburg: Metropolis.

Priddat, B. P. (2013) Moral führt! Moralische Deutungsmacht im ökonomischen Raum, Hamburg: Murmann Verlag.

Resick, C.J./Martin, G. S./Keating, M. A./Dickson, M. W./ Kwan, H. K./Peng, C. (2011): What Ethical Leadership Means to Me: Asian, American, and European Perspectives, in: Journal of Business Ethics, Vol. 101/No. 3, 435-457.

Retzmann, T. (1994): Wirtschaftsethik und Wirtschaftspädagogik. Eine fachdidaktische Analyse von Möglichkeiten zur Förderung der moralischen Urteils- und Handlungskompetenz von Führungskräften. Dissertationsschrift, Universität Köln.

Retzmann, T. (2005): Wirtschafts- und Unternehmensethik in den Lernfeldern der Berufsschule, in: Wirtschafts- und Unternehmensethik in der Aus- und Weiterbildung, in: Fritsche, G./Schmidt, M./Stöber, S. (Eds.): Speyrer Texte aus der Evangelischen Akademie der Pfalz, Bd. 12, 8-15.

Retzmann, T. (2009): Wirtschafts- und Unternehmensethik in der ökonomischen und politischen Bildung - Curriculumentwicklung im Projekt »ethos«, in: Politisches Lernen, Jg. 27/Heft 1/2,87-94.

Retzmann, T./Grammes, T. (2011): Wirtschafts- und Unternehmensethik in der ökonomischen und politischen Bildung. Ein fachdidaktisches Projekt zur Entwicklung exemplarischer Curriculumbausteine, in: Unterricht Wirtschaft + Politik, Jg. 1/Heft 2, 53-54.

Sadler-Smith, E. (2012): Before Virtue. Biology, Brain, Behavior, and the "Moral Sense", in: Business Ethics Quaterly, Vol. 22/No. 2, 351-375.

Saluador, R./Folger, R. G. (2009): Business Ethics and the Brain, in: Business Ethics Quarterly, Vol. 19/No. 1, 1-31.

Schlink, B. (2009): Das Moralische versteht sich nicht von selbst, in: Merkur - Deutsche Zeitschrift für europäisches Denken, Heft 722, 557-569.

Schwalbach, J./Klink, D. (2012): Der Ehrbare Kaufmann als individuelle Verantwortungskategorie der CSR-Forschung, in: Schneider, A./Schmitdpeter, R. (Hrsg.): Corporate Social Responsibility. Verantwortungsvolle Unternehmensführung in Theorie und Praxis, Berlin: Springer Verlag, 219-240.

Selznick, P. (1957): Leadership in Administration. A Sociological Interpretation, New York: Harper \& Row. 
Smith, C. N./Simpson, S. S./Huang, C. (2007): Why Managers Fail to Do the right Thing: An Empirical Study of Unethical and Illegal Conduct, in: Business Ethics Quarterly, Vol. 17/No. 4, 633-667.

Steinmann, H./Löbr, A. (1994): Grundlagen der Unternehmensethik, 2. Auflage, Stuttgart: Schäffer-Poeschel.

Stone, A. G./Russell, R. F./Patterson, K. (2004): Transformational Versus Servant Leadership. A Difference in Leader Focus, in: Leadership \& Organization Development Journal, Vol. 25/No. 4, 349-361.

The State Bar of California (TSBOC) (2013): Ethics. Link: http://ethics.calbar.ca.gov (zuletzt abgerufen am 03.08.2013).

Treviño, L. K. (1992): Moral Reasoning and Business Ethics: Implications for Research, Education, and Management,in: Journal of Business Ethics, Vol. 11/5-6, 445-459.

Treviño, L. K./Weaver, G. R./Gibson, D. G./Toffler, B. L. (1999): Managing Ethics and Legal Compliance. What Works and What Hurts in: California Management Review, Vol. 41/2, 131-151.

Treviño, L. K./Weaver, G. R./Reynolds, S.J. (2006): Behavioral Ethics in Organizations: A Review, in: Journal of management, Vol. 32/No. 6, 951-990.

Ulrich, P. (2008): Integrative Wirtschaftsethik. Grundlagen einer lebensdienlichen Ökonomie, 4. Auflage, Bern: Haupt.

U.S. Citizenship and Immigration Services (USCIS) (2013): \$ Sec. 316.10 Good moral character. Link: http://www.uscis.gov/iframe/ilink/docView/SLB/HTML/SLB/0-0-0-1/0-00-11261/0-0-0-30960/0-0-0-31086.html (zuletzt abgerufen am 03.08.2013).

Veiga, J. F./Golden, T. D./Decbant, K. (2004): Why Managers bend Company Rules, in: Academy of Management Executive, Vol. 18/No. 2, 84-90.

Walumbwa, F. O./Wernsing, T. (2012): From Transactional and Transformational Leadership to Authentic Leadership, in: Rumsey, M. G. (Ed.): The Oxford Handbook of Leadership, Oxford: Oxford University Press, 392-401.

Weaver, G. R./Treviño, L. K. (1999): Compliance and Values Oriented Ethics Programs. Influences on Employees' Attitudes and Behavior, in: Business Ethics Quarterly, Vol 9/No. 2, 315-335.

Werbane, P. (1999): Moral imagination and Management Decision-Making, Oxford: Oxford University Press.

Werbane, P. (2007): Moral Imagination and the Search for Ethical Decision-making in Management, in: Singer, A. E. (Ed.): Business Ethics and Strategy, Band 2, Farnham: Ashgate, 323-346.

Werhane, P./Freeman, E. R. (2006) (Ed.): The Blackwell Encyclopedia of Management, Volume 2, 2. Auflage, Malden: Wiley-Blackwell.

Wieland, J. (Hrsg.) (2004): Handbuch Wertemanagement, Marburg: Metropolis.

Wieland, J. (2005): Normativität und Governance, Marburg: Metropolis.

Wieland, J. (2007/1999): Die Ethik der Governance, 5. Auflage, Marburg: Metropolis.

Wieland, J. (Hrsg.) (2010a): Behavioural Business Ethics. Psychologie, Neuroökonomik und Governanceethik, Marburg: Metropolis.

Wieland, J. (2010b): Die Psychologie der Compliance - Motivation, Wahrnehmung und Legitimation von Wirtschaftskriminalität., in: Wieland, J./Steinmeyer, R./Grüninger, S. (Hrsg.): Handbuch Compliance Management, Berlin: ESV, 71-88.

Wieland, J. (2010c): Compliance Management als Corporate Governance - konzeptionelle Grundlagen und Erfolgsfaktoren, in: Wieland, J./Steinmeyer, R./Grüninger, S. (Hrsg.): Handbuch Compliance Management, Berlin: ESV, 15-38. 
Wieland, J. (2010d): Globales Wirtschaftsethos als transkulturelles Management, in: Küng, H./Leisinger, K./Wieland, J. (Hrsg.): Manifest Globales Wirtschaftsethos, München: dtv, 76-91.

Wieland, J. (2010e): Wie kann man Werte managen?, in: Küng, H./Leisinger, K./Wieland, J. (Hrsg.): Manifest Globales Wirtschaftsethos, München: dtv, 92-139.

Wieland, J. (2011): Wertemanagement, in: ABländer, Michael (Hrsg.): Handbuch der Wirtschafts- und Unternehmensethik, Stuttgart: Metzler, 245-252.

Wieland, J. (2013): Governancestrukturen des Gemeinwohls. Eine wirtschaftsethische Skizze, in: Kempf, E./Lüderssen, K./Volk, K. (Hrsg.): Gemeinwohl im Wirtschaftsstrafrecht, Berlin: De Gruyter.

Wieland, J. (2014a): The Ethics of Governance of Economic Organization, Berlin: Springer.

Wieland, J. (2014b): Integritäts- und Compliance Management als Corporate Governance konzeptionelle Grundlagen und Erfolgsfaktoren, in: Wieland, J./Steinmeyer, R./Grüninger, S. (Hrsg.): Handbuch Compliance Management, 2. Auflage, Berlin: ESV.

Zsolnai, L. (Ed.) (2013): Handbook of Business Ethics. Ethics in the New Economy, Oxford: Peter Lang. 